\title{
Generalized neighbor-interaction models induced by nonlinear lattices
}

\author{
F. Kh. Abdullaev, ${ }^{1, *}$ Yu. V. Bludov, ${ }^{2, \dagger}$ S. V. Dmitriev, ${ }^{3, \ddagger}$ P. G. Kevrekidis, ${ }^{4, \S}$ and V. V. Konotop ${ }^{2,5, \|}$ \\ ${ }^{1}$ Instituto de Física Teórica, UNESP, Rua Pamplona, 145, Sao Paulo, Brazil \\ ${ }^{2}$ Centro de Física Teórica e Computacional, Universidade de Lisboa, Complexo Interdisciplinar, Avenida Professor Gama Pinto 2 , \\ Lisboa 1649-003, Portugal \\ ${ }^{3}$ General Physics Department, Altai State Technical University, 656038 Barnaul, Russia \\ ${ }^{4}$ Department of Mathematics and Statistics, University of Massachusetts, Amherst, Massachusetts 01003, USA \\ ${ }^{5}$ Departamento de Física, Universidade de Lisboa, Campo Grande, Ed. C8, Piso 6, Lisboa 1749-016, Portugal
}

(Received 13 July 2007; published 23 January 2008)

\begin{abstract}
It is shown that the tight-binding approximation of the nonlinear Schrödinger equation with a periodic linear potential and periodic in space nonlinearity coefficient gives rise to a number of nonlinear lattices with complex, both linear and nonlinear, neighbor interactions. The obtained lattices present nonstandard possibilities, among which we mention a quasilinear regime, where the pulse dynamics obeys essentially the linear Schrödinger equation. We analyze the properties of such models both in connection to their modulational stability, as well as in regard to the existence and stability of their localized solitary wave solutions.
\end{abstract}

DOI: 10.1103/PhysRevE.77.016604

PACS number(s): 05.45.Yv

\section{INTRODUCTION}

It is generally recognized that mapping of a nonlinear evolution problem, described by a partial differential equation, into a simplified lattice, representing a set of coupled ordinary differential equations, appears to be a useful tool either for numerical (or semianalytical in the appropriate limits) study of the dynamics or for bringing intuitive understanding of the factors dominating the behavior of the systems. Examples of such an approach are well known for a long time in solid state physics [1] (e.g., the description of an electron in a crystal in the tight-binding approximation), in optics [2] (e.g., the description of the electric field in arrays of waveguides $[3,4]$ ), and more recently in the theory of matter waves in optical lattices (i.e., to the mean-field theory of arrays of Bose-Einstein condensates, which are described by the order parameter, rather than by the density matrix; this rules out the problem of ordering of field operators highly relevant to the case where each array contains only a few atoms, see, e.g., [5,6] for relevant reviews). In all the above cases, the periodicity is typically associated with the linear properties of the system and the respective dynamics is approximately described by the discrete nonlinear Schrödinger (DNLS) equation. The properties, relevance, and applications of the DNLS equation in diverse physical problems were analyzed in a variety of recent reviews $[7,8]$. We also note in passing that there has been a wide range of recent experimental works (and related theoretical works) associated with this prototypical DNLS equation including, e.g., the existence of Peierls-Nabarro barriers for discrete solitons [9], the modulational instability of uniform states both in optical experiments [10] and in BEC theory [11] and experi-

\footnotetext{
*fatkh@uzsci.net

†bludov@cii.fc.ul.pt

‡dmitriev.sergey.v@gmail.com

$\S$ kevrekid@math.umass.edu

"konotop@cii.fc.ul.pt
}

ments [12], the examination of multicomponent DNLS lattices [13], the study of discrete surface solitons [14] in one or two dimensions, and the formation of discrete vortices [15] in two dimensions, among many others.

On the other hand, there has recently been an increasing interest in studying nonlinear models, where the nonlinearity is also periodically modulated in space. Applications of such models extend from the propagation of electromagnetic waves in stratified media [16,17] to condensates of bosons [18] and condensates of boson-fermion mixtures [19] in optical lattices. It turned out that the spatially dependent nonlinearity may dramatically change properties of the system, in particular, the regions of existence of coherent localized structures and especially their corresponding stability properties.

Natural questions that arise in this context concern the mapping of the respective evolution equation into nonlinear lattice dynamics and the description of the existence, stability, and dynamical properties of such a lattice. In the present paper we consider both of these issues. While the first issue is technical and can be straightforwardly addressed by means of the Wannier function expansion, as it was suggested in [20], the study of the properties of the emerging lattices is a much richer problem in the present setting; its richness stems from the fact that the spatially dependent nonlinearity gives rise to complex nonlinear intersite interactions. Such forms of nonlinearity as the ones extracted below can significantly change the dynamical properties of the discrete system as it was shown in earlier research devoted to the spin waves in magnetic systems [21], to electromagnetic waves in waveguide arrays [22,23], and to arrays of Bose-Einstein condensates [24]. In the above mentioned studies of nonlinear lattices, however, one common feature was of crucial importance- that was the presence of dominant (or at least significant) on-site nonlinearity, which, e.g., in the case of a BEC loaded in an optical lattice is typically about two orders of magnitude larger than the hopping nonlinearity. In the present paper, we systematically derive and consider a far more general class of lattice evolution equations, including the cases where the on-site nonlinearity is exactly zero. 
It should be noted that such an analysis of reduced discrete models may prove particularly valuable in understanding either analytically or numerically the properties (especially the existence and stability) of solutions in the original partial differential equation (PDE) models from which the discrete ones arise. More specifically, in the discrete models, it is not only simpler and more inexpensive to perform numerical computations, but it is often possible to completely classify their solutions (see, e.g., for the prototypical example of the DNLS lattice the work of [25]) and their corresponding stability (see, e.g., for the same lattice, the work of [26]). This allows us to infer useful conclusions for the corresponding solutions in the original PDE.

The organization of the paper is as follows. In Sec. II we deduce the relevant lattice dynamical models with intersite nonlinearity starting with the evolution equation of the nonlinear Schrödinger (NLS) type with a spatially periodic potential and periodic nonlinearity. In Sec. III, we conduct the modulational stability analysis of the derived models. In Secs. IV and V, we examine the respective dynamical properties of the derived quasilinear and nonlinear models, while Sec. VI summarizes our findings and presents our conclusions, as well as directions of potential future interest.

\section{MODEL EQUATIONS}

\section{A. One-band approximation}

We start with the one-dimensional NLS equation

$$
i \frac{\partial \psi}{\partial t}=-\frac{\partial^{2} \psi}{\partial x^{2}}+\mathcal{U}(x) \psi+\mathcal{G}(x)|\psi|^{2} \psi
$$

where $\mathcal{U}(x)$ and $\mathcal{G}(x)$ are the coordinate-dependent linear and nonlinear potentials, respectively, both considered to be $\pi$-periodic functions: $\mathcal{U}(x)=\mathcal{U}(x+\pi)$ and $\mathcal{G}(x)=\mathcal{G}(x+\pi)$. We concentrate on the cases where the linear potential is an even function $\mathcal{U}(x)=\mathcal{U}(-x)$, while the nonlinearity may be either even or odd: $\mathcal{G}(x)=\sigma \mathcal{G}(-x)$ (hereafter $\sigma= \pm 1$ ). It is important to point out here that the starting point of Eq. (1) implies the fact that we operate within the mean-field limit (relevant to temperatures very close to $T=0$ ) and the superfluid regime of the condensate; see $[5,6]$ for more details.

In order to map Eq. (1) into a lattice equation we follow [20]. To this end, we introduce the linear eigenvalue problem

$$
-\frac{d^{2} \varphi_{\alpha q}(x)}{d x^{2}}+\mathcal{U}(x) \varphi_{\alpha q}(x)=\mathcal{E}_{\alpha q} \varphi_{\alpha q}(x),
$$

where $\varphi_{\alpha q}(x)$ is a Bloch function, and $\alpha \geqslant 1$ and $q$ stand for the band number and for the Bloch wavenumber in the first Brillouin zone: $q \in[-1,1]$, and define the Wannier functions

$$
w_{n \alpha}(x)=\frac{1}{\sqrt{2}} \int_{-1}^{1} \varphi_{\alpha q}(x) e^{-i \pi n q} d q,
$$

which constitute an orthonormal set of real and exponentially decaying functions [27].

We seek the solution of Eq. (1) in the form of a series over the full orthonormal set of Wannier functions

$$
\psi(x, t)=\sum_{n, \alpha} c_{n \alpha}(t) w_{n \alpha}(x) .
$$

For the next consideration we notice that the Wannier functions of the $\alpha$ th band possess either even or odd parity $w_{0 \alpha}(x)=(-1)^{1+\alpha} w_{0 \alpha}(-x)$ and are characterized by the property $w_{n \alpha}(x)=w_{0 \alpha}(x-n \pi)$.

Now we make the most crucial approximation of our model, namely, that the continuum Eq. (1) can be accurately described within the one-band approximation. As it was shown in [20], this assumption fails to describe the original continuous model when one studies dynamical processes associated with the generation of the frequencies belonging to the higher bands. However, it is reasonably accurate in describing static solutions (in particular, localized modes) as well as their stability. Also, the lattices of generalized neighbor interactions derived below within the framework of the one-band approximation, are of interest in their own right, per their particularities and differences in comparison to other models of similar type; cf. [22,23].

We thus assume that only one band, say the $\alpha$ th one, is populated. Now, substituting the expansion (4) in Eq. (1) we arrive at the equation (see [5,20] for more details)

$$
\begin{aligned}
i \dot{c}_{n \alpha} & -c_{n \alpha} \omega_{0 \alpha}-\left(c_{n-1, \alpha}+c_{n+1, \alpha}\right) \omega_{1 \alpha} \\
& -\sum_{n_{1}, n_{2}, n_{3}} c_{n_{1} \alpha} \bar{c}_{n_{2} \alpha} c_{n_{3} \alpha} W_{\alpha \alpha \alpha \alpha}^{n_{1} n_{2} n_{3}}=0,
\end{aligned}
$$

where

$$
W_{\alpha \alpha_{1} \alpha_{2} \alpha_{3}}^{n n_{1} n_{2} n_{3}}=\int_{-\infty}^{\infty} \mathcal{G}(x) w_{n \alpha}(x) w_{n_{1} \alpha_{1}}(x) w_{n_{2} \alpha_{2}}(x) w_{n_{3} \alpha_{3}}(x) d x
$$

are the nonlinear overlap integrals, and $\omega_{n \alpha}$ are the coefficients of the Fourier series expansion of the eigenvalue $\mathcal{E}_{\alpha q}$ as follows:

$$
\mathcal{E}_{\alpha q}=\sum_{n} \omega_{n \alpha} e^{i \pi n q}, \quad \omega_{n \alpha}=\frac{1}{2} \int_{-1}^{1} \mathcal{E}_{\alpha q} e^{-i \pi n q} d q .
$$

The overbar stands for complex conjugation, and an overdot stands for the derivative with respect to time. In Eq. (5) we have taken into account that in a general situation, for a periodic potential $\mathcal{U}(x)$ of rather large amplitude, the lowest bands are very narrow, and hence the Fourier coefficients $\omega_{n \alpha}$ decay rapidly with increasing $n$ (so that $\left|\omega_{0 \alpha}\right| \gg\left|\omega_{1 \alpha}\right|$ $\left.\gg\left|\omega_{2 \alpha}\right|\right)$, which, in turn, allows us to neglect the coefficients $\omega_{n \alpha}$ with $n \geqslant 2$.

For the lowest bands the Wannier functions are well localized on the scale of one lattice period (and can be reasonably well approximated by the eigenstates of the linear oscillator) and thus the overlap integrals involving next-nearest neighbors (i.e., lattice minima separated by two lattice maxima) are negligibly small. This allows us to drop also the terms involving $W_{\alpha_{1} \alpha_{2} \alpha_{3} \alpha_{4}}^{n_{1} n_{2} n_{3} n_{4}}$ with at least one pair of the upper indices satisfying $\left|n_{j}-n_{k}\right| \geqslant 2$. We, however, emphasize that it is of crucial importance to leave the nonlinear terms with hopping between the neighbor sites, which for specific choices of the nonlinear interactions $\mathcal{G}(x)$ can be comparable 
with or even stronger than the on-site nonlinearity (see below).

Now we use the symmetry of the integrals $W$ with respect to permutations of the indices and introduce

$$
\begin{gathered}
W_{0}=W_{\alpha \alpha \alpha \alpha}^{n n n n}=W_{\alpha \alpha \alpha \alpha \alpha}^{0000}, \\
W_{1}=W_{\alpha \alpha \alpha \alpha}^{n, n-1, n-1, n-1}=\sigma W_{\alpha \alpha \alpha \alpha}^{n, n, n, n-1}=W_{\alpha \alpha \alpha \alpha \alpha}^{1000}, \\
W_{2}=W_{\alpha \alpha \alpha \alpha}^{n, n, n-1, n-1}=W_{\alpha \alpha \alpha \alpha \alpha}^{1100},
\end{gathered}
$$

where $\sigma=1$ and $\sigma=-1$ for $\mathcal{G}(x)$ even and odd and

$$
W_{j}=\int_{-\infty}^{\infty} \mathcal{G}(x) w_{1 \alpha}^{j}(x) w_{0 \alpha}^{4-j}(x) d x, \quad j=0,1,2 .
$$

In the case of odd nonlinearity $(\sigma=-1)$ the terms $W_{0}$ and $W_{2}$ are always equal to zero due to the fact that the integrand in Eq. (9) is odd with respect to the points $X=0$ and $X=\pi / 2$, correspondingly. We thus arrive at the equation

$$
\begin{aligned}
i \dot{c}_{n}= & \omega_{0} c_{n}+\omega_{1}\left(c_{n-1}+c_{n+1}\right)+W_{0}\left|c_{n}\right|^{2} c_{n}+W_{1}\left(\left|c_{n-1}\right|^{2} c_{n-1}\right. \\
& +\sigma \bar{c}_{n-1} c_{n}^{2}+2 \sigma\left|c_{n}\right|^{2} c_{n-1}+2\left|c_{n}\right|^{2} c_{n+1}+\bar{c}_{n+1} c_{n}^{2} \\
& \left.+\sigma\left|c_{n+1}\right|^{2} c_{n+1}\right)+W_{2}\left(2\left|c_{n-1}\right|^{2} c_{n}+\bar{c}_{n} c_{n-1}^{2}+\bar{c}_{n} c_{n+1}^{2}\right. \\
& \left.+2\left|c_{n+1}\right|^{2} c_{n}\right),
\end{aligned}
$$

where we have dropped the zone index $\alpha$ (e.g., $c_{n, \alpha}$ is redefined as $c_{n}$, etc.)

Equation (10) is the main discrete model studied in the present paper. We notice that it has a Hamiltonian structure: $i \dot{c}_{n}=\partial H / \partial \bar{c}_{n}$ with the Hamiltonian

$$
\begin{aligned}
H= & \sum_{n}\left[\omega_{0}\left|c_{n}\right|^{2}+\omega_{1}\left(c_{n-1} \bar{c}_{n}+\bar{c}_{n-1} c_{n}\right)+\frac{W_{0}}{2}\left|c_{n}\right|^{4}+W_{1}\left(\left|c_{n-1}\right|^{2}\right.\right. \\
& \left.+\sigma\left|c_{n}\right|^{2}\right)\left(c_{n} \bar{c}_{n-1}+\bar{c}_{n} c_{n-1}\right)+2 W_{2}\left|c_{n-1}\right|^{2}\left|c_{n}\right|^{2}+\frac{W_{2}}{2}\left(c_{n}^{2} \bar{c}_{n-1}^{2}\right. \\
& \left.\left.+\bar{c}_{n}^{2} c_{n-1}^{2}\right)\right]
\end{aligned}
$$

and with the standard Poisson brackets. Another integral of motion is the sum $N=\Sigma_{n}\left|c_{n}\right|^{2}$, reflecting the conservation of the "number of atoms" (in keeping with the BEC motivation of our analysis) of the original Eq. (1).

\section{B. Particular cases}

So far, we have established that the spatial dependence of the nonlinearity plays a critical role in determining the values of the nonlinear coefficients and thus accordingly determines the type of the discrete equation (10). Now we consider a number of particular cases. First of all, however, we notice that in the case of odd nonlinearity function, i.e., for $\sigma=-1$, neither purely even $\left(c_{n}=c_{-n}\right)$, nor purely odd $\left(c_{n}=-c_{-n}\right)$ solutions can exist. This follows directly from the symmetry of Eq. (10) [or Eq. (1)].

For illustration of the localized solutions we use the potential $\mathcal{U}(X)=-3 \cos (2 X)$ (both for the detailed calculations of this section, and for the numerical simulations of the fol- lowing sections). Also we restrict our considerations to the first band, i.e., we take $\alpha=1$. In that case, the linear overlap coefficients for the first band are computed as $\omega_{0} \approx-0.839$, $\omega_{1} \approx-0.051$, and $\omega_{2} \approx 0.002$ [37].

One can distinguish five cases as follows:

Case 1. $W_{0}=W_{1}=W_{2}=0$. This is a quasilinear case, which is made possible, for example, by an odd nonlinearity $(\sigma=-1)$ of the form

$$
\mathcal{G}(x)=\sin (2 x)-1.3706 \sin (4 x)
$$

(obviously the number of possible realizations of this and other cases reported below is naturally unlimited). Our approach both in this example and below is motivated by the nature of the lattice that we wish to construct (i.e., by the type of overlap integral that we wish to preserve or eliminate). For instance, in this example, the odd nonlinearity guarantees that $W_{0}=W_{2}=0$, while the expression of Eq. (12) uses one tunable parameter (the amplitude of the second harmonic) to achieve $W_{1}=0$.

Case 2. $W_{0}=W_{2}=0, W_{1} \neq 0$, is achieved, e.g., by choosing

$$
\mathcal{G}(x)=10 \sin (2 x)
$$

$(\sigma=-1)$. Now the lattice model (10) is reduced to

$$
\begin{aligned}
i \dot{c}_{n}= & \omega_{0} c_{n}+\omega_{1}\left(c_{n-1}+c_{n+1}\right)+W_{1}\left(\left|c_{n-1}\right|^{2} c_{n-1}-\bar{c}_{n-1} c_{n}^{2}\right. \\
& \left.-2\left|c_{n}\right|^{2} c_{n-1}+2\left|c_{n}\right|^{2} c_{n+1}+\bar{c}_{n+1} c_{n}^{2}-\left|c_{n+1}\right|^{2} c_{n+1}\right),
\end{aligned}
$$

and in the case at hand $W_{1} \approx 0.045$.

Alternatively, this case can be realized by choosing even nonlinearity $(\sigma=1)$

$$
\mathcal{G}(x)=0.0275+4.809 \cos (2 x)-10 \cos (4 x) .
$$

Now $W_{1} \approx 0.012$ and the lattice equation reads

$$
\begin{aligned}
i \dot{c}_{n}= & \omega_{0} c_{n}+\omega_{1}\left(c_{n-1}+c_{n+1}\right)+W_{1}\left(\left|c_{n-1}\right|^{2} c_{n-1}+\bar{c}_{n-1} c_{n}^{2}\right. \\
& \left.+2\left|c_{n}\right|^{2} c_{n-1}+2\left|c_{n}\right|^{2} c_{n+1}+\bar{c}_{n+1} c_{n}^{2}+\left|c_{n+1}\right|^{2} c_{n+1}\right) .
\end{aligned}
$$

Case 3. $W_{0}=W_{1}=0, W_{2} \neq 0$ is obtained, for instance, for the even nonlinearity $(\sigma=1)$

$$
\mathcal{G}(x)=-23.836+48.882 \cos (2 x)-37.778 \cos (4 x),
$$

for which $W_{2} \approx-0.0136$. The model (10) is now simplified as

$$
\begin{aligned}
i \dot{c}_{n}= & \omega_{0} c_{n}+\omega_{1}\left(c_{n-1}+c_{n+1}\right)+W_{2}\left(2\left|c_{n-1}\right|^{2} c_{n}+\bar{c}_{n} c_{n-1}^{2}+\bar{c}_{n} c_{n+1}^{2}\right. \\
& \left.+2\left|c_{n+1}\right|^{2} c_{n}\right) .
\end{aligned}
$$

Case $4 . W_{0}=0, W_{1,2} \neq 0$ can be achieved by using an even nonlinearity $(\sigma=1)$

$$
\mathcal{G}(x)=7.795-10 \cos (2 x) .
$$

Now the overlap integrals are as follows: $W_{1} \approx 0.0148$ and $W_{2} \approx 0.0045$. The lattice model then reads

$$
\begin{aligned}
i \dot{c}_{n}= & \omega_{0} c_{n}+\omega_{1}\left(c_{n-1}+c_{n+1}\right)+W_{1}\left(\left|c_{n-1}\right|^{2} c_{n-1}+\bar{c}_{n-1} c_{n}^{2}\right. \\
& \left.+2\left|c_{n}\right|^{2} c_{n-1}+2\left|c_{n}\right|^{2} c_{n+1}+\bar{c}_{n+1} c_{n}^{2}+\left|c_{n+1}\right|^{2} c_{n+1}\right) \\
& +W_{2}\left(2\left|c_{n-1}\right|^{2} c_{n}+\bar{c}_{n} c_{n-1}^{2}+\bar{c}_{n} c_{n+1}^{2}+2\left|c_{n+1}\right|^{2} c_{n}\right) .
\end{aligned}
$$


Case 5. $\left|W_{1}\right|,\left|W_{2}\right| \ll\left|W_{0}\right|$ is the standard case of the onsite nonlinearity (the DNLS equation)

$$
i \dot{c}_{n}=\omega_{0} c_{n}+\omega_{1}\left(c_{n-1}+c_{n+1}\right)+W_{0}\left|c_{n}\right|^{2} c_{n} .
$$

This form of the lattice dynamical model is obtained for generic nonlinearities, Eq. (21) is well studied in the literature (see, e.g., [7]) and that is why it will not be addressed in this paper.

\section{MODULATIONAL INSTABILITY}

As is customary we start with the analysis of the modulational instability of plane-wave solutions of Eq. (10) (for a general study of the modulational instability of the planewave background in the DNLS-type equations, see, e.g., [28-30]). This stability analysis is performed not only because it is of interest in its own right but also because the solitary wave solutions that we plan on constructing for the above presented lattices should be produced on a dynamically stable background. Using the plane-wave solution of the form

$$
c_{n}=F e^{i(q n-\omega t)},
$$

where $F$ is a constant amplitude, we obtain the dispersion relation (in the absence of the previously considered cubic onsite terms)

$$
\begin{aligned}
\omega= & \omega_{0}+2 \omega_{1} \cos (q)+4 W_{1} F^{2}(\sigma+1) \cos (q)+2 W_{2} F^{2}[2 \\
& +\cos (2 q)] .
\end{aligned}
$$

To examine the linear stability, we perturb the plane-wave solution in the form

$$
c_{n}=\left(F+A e^{i(Q n-\Omega t)}+\bar{B} e^{-i(Q n-\Omega t)}\right) e^{i(q n-\omega t)},
$$

with $|A|,|B| \ll|F|$ and linearize with respect to $A$ and $B$. As a result we obtain two branches of the linear excitations $\Omega_{1,2}(Q)$,

$$
\Omega_{1,2}=M_{-} \pm \sqrt{\left(M_{+}-D\right)^{2}-F^{4} \Delta}
$$

where

$$
\begin{gathered}
D=\omega-\omega_{0}-4 W_{1}(1+\sigma) F^{2} \cos (q)-4 W_{2} F^{2}[\cos (Q)+1], \\
M_{-}=-2\left[\omega_{1}+2 W_{1}(1+\sigma) F^{2}\right] \sin (Q) \sin (q) \\
-4 W_{2} F^{2} \sin (Q) \sin (2 q), \\
M_{+}=2\left[\omega_{1}+2 W_{1}(1+\sigma) F^{2}\right] \cos (Q) \cos (q) \\
+4 W_{2} F^{2} \cos (Q) \cos (2 q), \\
\Delta=4\left\{W_{1}(1+\sigma)[1+\cos (Q)] \cos (q)+W_{2}[2 \cos (Q)\right. \\
+\cos (2 q)]\}^{2}+4(1-\sigma)^{2} \sin ^{2}(q) W_{1}^{2}[1-\cos (Q)]^{2} .
\end{gathered}
$$

Let us consider different special cases for $\sigma=-1$ (the case $\sigma=1$ is investigated in [22]).

(a) Homogeneous background is described by $q=0$. Now the two branches of the solutions collapse and the dispersion relation acquires the form

$$
\begin{aligned}
\Omega^{2}= & 16\left(\omega_{1}+2 W_{2} F^{2}\right) \sin ^{2}\left(\frac{Q}{2}\right)\left[\left(\omega_{1}+6 W_{2} F^{2}\right) \sin ^{2}\left(\frac{Q}{2}\right)\right. \\
& \left.-3 W_{2} F^{2}\right] .
\end{aligned}
$$

The carrier field is stable if and only if the two following conditions are satisfied:

$$
\begin{gathered}
\left(\omega_{1}+2 W_{2} F^{2}\right) W_{2} \leqslant 0 \\
\left(\omega_{1}+2 W_{2} F^{2}\right)\left(\omega_{1}+3 W_{2} F^{2}\right) \geqslant 0,
\end{gathered}
$$

where the first of these conditions demands non-negativity of the coefficient $k$ in the expansion of Eq. (26) of the form of $\Omega^{2}(Q)=k Q^{2}$, which is valid for small $Q$. The second condition demands non-negativity of $\Omega^{2}(Q)$ at the zone boundary, $Q=\pi$.

We point out that the long wavelength excitations' group velocity dispersion is given by

$$
\left.\frac{\partial^{2} \Omega}{\partial Q^{2}}\right|_{Q=0}=2 W_{2}\left[2 W_{2} F^{4}+\left(8 W_{2}-6+\omega_{1}\right) F^{2}+4 \omega_{1}\right],
$$

and takes zero values for

$$
F=\left\{\frac{6-\omega_{1}-8 W_{2}}{4 W_{2}} \pm \sqrt{\left(\frac{6-\omega_{1}-8 W_{2}}{4 W_{2}}\right)^{2}-\frac{2 \omega_{1}}{W_{2}}}\right\}^{1 / 2}
$$

This last condition determines the domain of the parameters where shock waves can be observed [31].

(b) Staggered background corresponds to $q=\pi$. The dispersion relation is

$$
\begin{aligned}
\Omega^{2}= & 16\left(\omega_{1}-2 W_{2} F^{2}\right) \sin ^{2}\left(\frac{Q}{2}\right)\left[\left(\omega_{1}-6 W_{2} F^{2}\right) \sin ^{2}\left(\frac{Q}{2}\right)\right. \\
& \left.+3 W_{2} F^{2}\right] .
\end{aligned}
$$

Similar to Eq. (27) we introduce stability criteria in the form

$$
\begin{gathered}
\left(\omega_{1}-2 W_{2} F^{2}\right) W_{2} \geqslant 0, \\
\left(\omega_{1}-2 W_{2} F^{2}\right)\left(\omega_{1}-3 W_{2} F^{2}\right) \geqslant 0 .
\end{gathered}
$$

Note that the dispersion relations for $q=0, \pi$ do not depend on the coefficient $W_{1}$.

(c) Phase alternating background where $q=\pi / 2$. The dispersion relation is

$$
\begin{aligned}
\Omega_{1,2}= & -2 \omega_{1} \sin (Q) \\
& \pm 4 \sqrt{2} F^{2} \sin \left(\frac{Q}{2}\right) \sqrt{\left(W_{2}^{2}+W_{1}^{2}\right) \cos (Q)-W_{1}^{2}} .
\end{aligned}
$$

The condition for the presence of modulational instability in this case is

$$
\cos (Q)<\frac{W_{1}^{2}}{W_{1}^{2}+W_{2}^{2}}
$$




\section{QUASILINEAR MODEL (CASE 1)}

Let us now turn to the quasilinear model of case 1 . We notice that the term "quasilinear" is used in order to emphasize the existence of solely higher order contributions from more distant neighbors which are small but not necessarily zero. This provides us with an excellent benchmark of our derivation since in the discrete linear case, the dynamical equation can be solved explicitly and subsequently compared to the full results of the original partial differential equation (for which the discrete model was developed as an approximation). In particular, it is well known that the linear discrete case, with a compactum of initial data $c_{n}(0)=A \delta_{n 0}$ has a solution of the form

$$
c_{n}(t)=A(-i)^{n} \exp \left(-i \omega_{0} t\right) J_{n}\left(2 \omega_{1} t\right),
$$

where $J_{n}$ is the Bessel function of order $n$.

We have tested this analytical prediction of the discrete model in the partial differential equation (1) with the "nonlinear potential" of Eq. (12). The results of our numerical simulations can be found in Fig. 1, which highlights the excellent agreement between the analytical and the numerical results. This also serves to showcase the accuracy of the reduction via the tight-binding approximation of the original partial differential equation. We will hereafter focus more on the details of these discrete models and of their solitary wave solutions.

The remarkable accuracy of the tight-binding model in the case at hand can be easily understood. Indeed, we are dealing with a perfect lattice (i.e., having no defects). Inter-band transitions, which are the cause of the failure of the one-band approximation when they exist, are only due to the nonlinear coupling of bands, and exactly this factor is anomalously small for the chosen nonlinearity. The first indication on this fact is given by the zero contributions of $W_{0,1,2}$. Next, due to the symmetry of the Wannier functions, and the symmetry of the nonlinearity, one concludes that $W_{\alpha \alpha \beta \beta}^{n n n n}=0$ for all $\alpha$ and $\beta$, i.e., there exists no tunneling between the same sites of two different bands. Even more generally (also due to the symmetry), $W_{\alpha, \beta, \beta, \alpha+2 \gamma}^{n, n-m, n+m, n}=0$ (for arbitrary integers $\beta, \gamma, n$, and $m$ ). For completeness we have checked the numerical values of the other interband overlap integrals for the three lowest bands. The integrals greater than 0.01 are as follows (the ones obtained by the symmetry reductions are not shown): $W_{2111}^{0000}=0.0465, W_{2221}^{0000}=-0.1360, W_{2211}^{1000}=-0.0228$, $W_{3111}^{1000}=-0.0432, W_{3311}^{1000}=-0.0301, W_{1333}^{1000}=-0.0117$. The coef ficients describing energy transfer from the first to the second and the third bands are $W_{2111}^{0000}=0.0465, W_{3111}^{0000}=0, W_{2111}^{1000}$ $=0.0077, \quad W_{3111}^{1000}=-0.0432, \quad W_{2111}^{1100}=0.0004$, and $W_{3111}^{1100}$ $=0.0011$, i.e., either have relatively small value or are identically zero. This explains the high accuracy of the one-band approximation.

Finally, we notice that in the case at hand the dispersion relation (25) takes the form independent of the wave amplitude $F$,

$$
\Omega= \pm 2 \omega_{1}[\cos (q)-\cos (Q \mp q)],
$$

which is the dispersion relation for linear phonons, and hence no instabilities can occur.
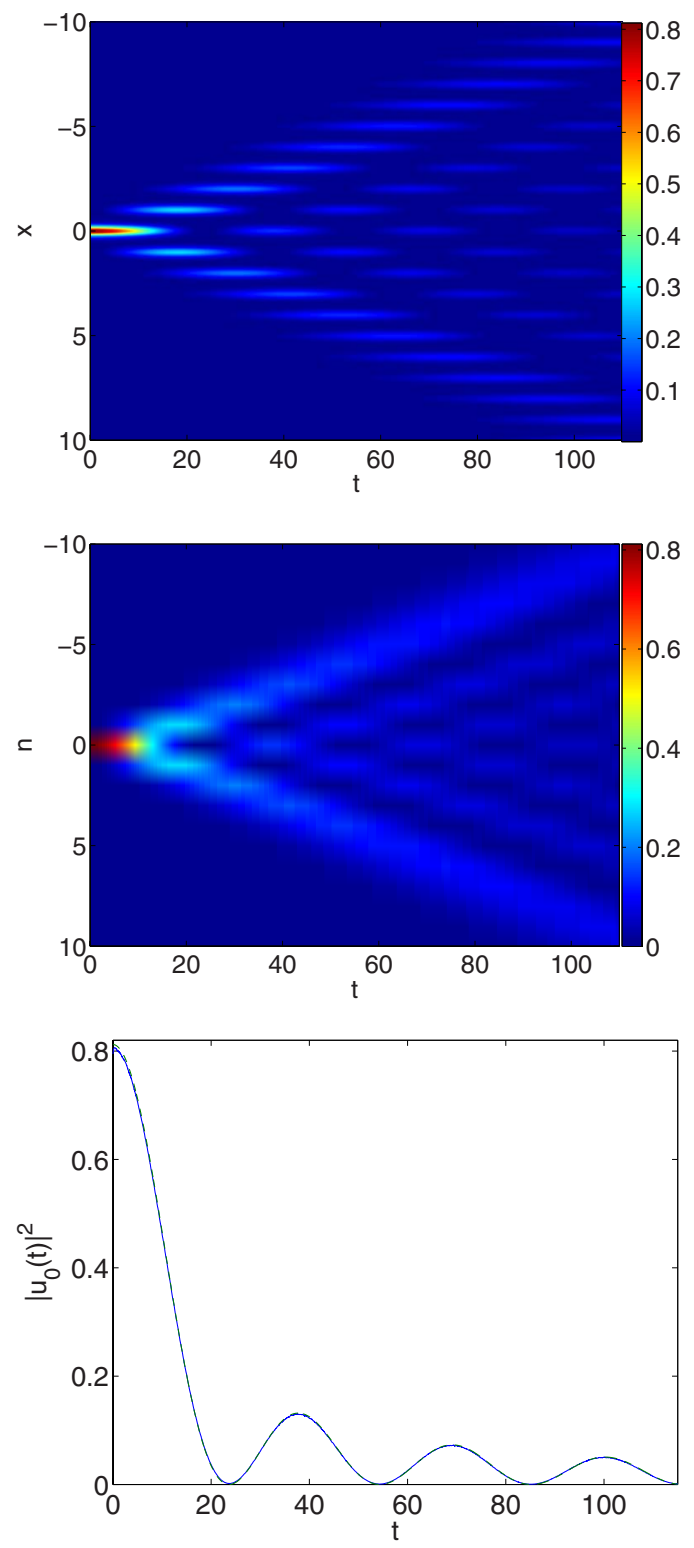

FIG. 1. (Color online) The first and second panels show the space-time contour plot of the solution in the PDE of Eq. (1) and the discrete model analytical prediction (33), respectively. In the latter case, space is normalized over the period of the linear potential, so that it can be compared with the lattice results. To accentuate the excellent agreement between analytical and numerical results, the third panel shows the time evolution of the amplitude at the central site of the configuration compared between the PDE numerical result (solid line) and the discrete equation analytical result of Eq. (33) (dashed line). The two are practically indistinguishable.

\section{NONLINEAR MODELS (CASES 2 AND 3)}

The above confirmation of the high accuracy of the tightbinding model for the description of the pulse dynamics motivated us to study the coherent structure solutions of Eq. (10) and their properties for cases 2 and 3 singled out in Sec. II B. It is convenient to join the equations of cases 2 and 3 and to consider for $\sigma=1$ the model 


$$
\begin{aligned}
i \dot{c}_{n}= & \omega_{0} c_{n}+\omega_{1}\left(c_{n-1}+c_{n+1}\right)+W_{1}\left(\left|c_{n-1}\right|^{2} c_{n-1}+\bar{c}_{n-1} c_{n}^{2}\right. \\
& \left.+2\left|c_{n}\right|^{2} c_{n-1}+2\left|c_{n}\right|^{2} c_{n+1}+\bar{c}_{n+1} c_{n}^{2}+\left|c_{n+1}\right|^{2} c_{n+1}\right) \\
& +W_{2}\left(2\left|c_{n-1}\right|^{2} c_{n}+\bar{c}_{n} c_{n-1}^{2}+\bar{c}_{n} c_{n+1}^{2}+2\left|c_{n+1}\right|^{2} c_{n}\right) .
\end{aligned}
$$

Let us seek stationary solutions of Eq. (35) of the form

$$
c_{n}(t)=f_{n} e^{-i \omega t},
$$

with real $f_{n}$. Using this ansatz, we obtain the equation for the amplitudes

$$
\begin{aligned}
& \left(\omega_{0}-\omega\right) f_{n}+\omega_{1}\left(f_{n-1}+f_{n+1}\right)+W_{1}\left[f_{n-1}^{3}+f_{n+1}^{3}\right. \\
& \left.\quad+3 f_{n}^{2}\left(f_{n+1}+f_{n-1}\right)\right]+3 W_{2} f_{n}\left(f_{n-1}^{2}+f_{n+1}^{2}\right)=0 .
\end{aligned}
$$

We attempt to find the pulse (bright soliton) and kink (dark soliton) solutions. Our strategy in searching for these solutions will be as follows. We will first formulate the necessary conditions for the existence of the soliton solutions considering their carrier constant-amplitude field and also the soliton tail solutions. This will narrow the domain of parameters where such solutions can be expected. Then, we will attempt to construct the desired soliton solutions and subsequently study their stability.

The staggered and nonstaggered stationary solutions are connected by the following symmetry relation [25]: if $f_{n}$ is a solution of Eq. (37) for definite $W_{1}, W_{2}$, and $\omega<\omega_{0}$ $\left(\omega>\omega_{0}\right)$, then $(-1)^{n} f_{n}$ is a solution for $\tilde{W}_{1}=W_{1}, \widetilde{W}_{2}=-W_{2}$, and $\tilde{\omega}=2 \omega_{0}-\omega>\omega_{0}\left(\tilde{\omega}<\omega_{0}\right)$. We also note that the stability analysis of any stationary solution can also be done, without loss of generality, for only, say, nonstaggered carrier field. This is so because the dynamics in the vicinity of the stationary solution is governed by Eq. (51) (see below), which is invariant with respect to the following transformation: $\epsilon_{n}$ $\rightarrow(-1)^{n} \epsilon_{n}, f_{n} \rightarrow(-1)^{n} f_{n}, \omega \rightarrow 2 \omega_{0}-\omega, W_{2} \rightarrow-W_{2}$, and $t \rightarrow$ $-t$. Bearing this in mind, in the following we will discuss only stationary solutions with a nonstaggered background.

\section{A. Constant-amplitude solution and soliton tails}

A pulse solution must satisfy the boundary conditions $\left|c_{n}\right|^{2} \rightarrow 0$ for $n \rightarrow \pm \infty$, while for the kink solution one must have $\left|c_{n}\right|^{2} \rightarrow F^{2}>0$ for $n \rightarrow \pm \infty$. Thus, the existence and stability of the carrying field solution, described by formula (22) with real $F$, is a necessary condition for the existence and stability of the soliton solutions.

One always has the trivial solution $F=0$ and from the expression (23) one can have two nonzero solutions with

$$
F^{2}=\frac{\omega-\omega_{0}-2 \omega_{1}}{8 W_{1}+6 W_{2}},
$$

if the expression in the right-hand side of Eq. (38) is positive. In the case $8 W_{1}+6 W_{2}=0, F$ can be arbitrary if $\omega=\omega_{0}+2 \omega_{1}$, but we will not study this very special case.

Substituting Eq. (38) into Eq. (25) we obtain the spectrum of the carrier field with $F^{2}>0$, whose stability criteria (in full analogy with Sec. III) are

$$
\begin{aligned}
& {\left[W_{1}\left(\omega-\omega_{0}+2 \omega_{1}\right)+W_{2}\left(\omega-\omega_{0}+\omega_{1}\right)\right]} \\
& \times\left(4 W_{1}+3 W_{2}\right)\left(\omega-\omega_{0}-2 \omega_{1}\right) \leqslant 0,
\end{aligned}
$$

$$
\begin{aligned}
& {\left[W_{1}\left(\omega-\omega_{0}+2 \omega_{1}\right)+W_{2}\left(\omega-\omega_{0}+\omega_{1}\right)\right]} \\
& \quad \times\left[2 W_{1}\left(\omega-\omega_{0}+2 \omega_{1}\right)+3 W_{2}\left(\omega-\omega_{0}\right)\right] \geqslant 0 .
\end{aligned}
$$

From the asymptotic properties mentioned above, one can express the soliton tails as

$$
f_{n} \sim F+\xi_{n}
$$

at $|n| \rightarrow \infty$, where small $\xi_{n}$ are real and are independent on $t$. Substituting Eq. (40) into Eq. (37) and linearizing with respect to $\xi_{n}$ one obtains

$$
\gamma \xi_{n-1}+\beta \xi_{n}+\gamma \xi_{n+1}=0
$$

with

$$
\begin{gathered}
\beta=\omega_{0}-\omega+12 W_{1} F^{2}+6 W_{2} F^{2}, \\
\gamma=\omega_{1}+6 W_{1} F^{2}+6 W_{2} F^{2} .
\end{gathered}
$$

Seeking solutions to Eq. (41) in the form

$$
\xi_{n} \sim C_{ \pm} z^{n}, \quad n \rightarrow \pm \infty,
$$

we come to the characteristic equation

$$
\gamma z^{2}+\beta z+\gamma=0
$$

Thus, $z$ is one of the roots $z_{1,2}$,

$$
z_{1}=\frac{1}{z_{2}}=-\frac{\beta}{2 \gamma}+\sqrt{\frac{\beta^{2}}{4 \gamma^{2}}-1},
$$

providing $\left|\xi_{n}\right| \rightarrow 0$ as $|n| \rightarrow \infty$.

For the soliton, which is either a homoclinic or a heteroclinic of the map, generated by Eq. (37), $\xi_{n} \equiv 0$ must be a hyperbolic point which happens only if the roots $z_{1,2}$ are real, i.e., if

$$
\beta^{2}-4 \gamma^{2}>0
$$

The sign of $z$ specifies the type of tail solution: $z>0$ corresponds to the tail decaying monotonically with distance from the soliton's center, while $z<0$ means that the decaying tail solution oscillates near the carrier solution $F$.

The absolute value of $z$ characterizes the degree of localization of the tail. If $|z|$ is small, then the tail solution is weakly localized, otherwise it is strongly localized. We found that, in many cases, there is a correlation between the degree of localization of the tail solution and that of the soliton itself.

The tail solution was found from the linearized equation and it can only provide necessary conditions for the existence of a soliton solution. The nonlinear terms, depending on their structure and the values of the corresponding coefficients, can either lead to unbounded solutions or to nonlocalized solutions, or to the desired bounded and localized soliton solutions.

Having a tail solution one can attempt to construct the corresponding soliton solution. To do so, we use the tail solution defined by Eq. (40) to set the initial values for $f_{n-1}$ and $f_{n}$ and then find $f_{n+1}$ from Eq. (37), solving the cubic (for $W_{2}=0$ ) or the quadratic (for $W_{1}=0$ ) algebraic equation. The proper choice of the integration constants $C_{ \pm}$systematically allows one to obtain equilibrium on-site or intersite soliton 
solutions, if they exist [38]. To conclude this section we summarize the necessary conditions for the existence of pulses and kinks.

Pulse in cases 2 and 3 . Since the carrying field for pulses with $F=0$ always exists, there remains only one necessary condition, namely, the condition of the existence of the tail solution of Eq. (45). For $F=0$ this condition is satisfied for any $W_{1}$ and $W_{2}$ and for both cases 2 and 3 when

$$
\begin{aligned}
& \omega>\omega_{0}-2 \omega_{1}=-0.73732741 \equiv \omega_{a}, \\
& \omega<\omega_{0}+2 \omega_{1}=-0.94041721 \equiv \omega_{b} .
\end{aligned}
$$

These conditions simply state that the localized pulses must be located outside the phonon band of the spectrum given by the interval $\left[\omega_{b}, \omega_{a}\right]$.

The necessary conditions of the existence of a kink include the condition of the existence of the carrier field with $F^{2}>0$, Eq. (38), the stability condition for the carrier field, Eq. (39), and condition (45) of the existence of the tail solution.

Kink in case 2. All three necessary conditions are satisfied when $W_{1}>0$ and $\omega_{b}<\omega<\omega_{a}$, and they are not satisfied simultaneously for $W_{1}<0$.

Kink in case 3 . All three necessary conditions are satisfied when $W_{2}>0$ and $\omega_{b}<\omega<\omega_{0}$, while for $W_{2}<0$ they are satisfied for $\omega_{0}<\omega<\omega_{a}$.

We also specify the frequencies at which $z$ changes sign. In case 2 the frequencies are

$$
\begin{aligned}
& \omega_{0}-(2 / 3) \omega_{1}=-0.80502401 \equiv \omega_{c}, \\
& \omega_{0}+(2 / 3) \omega_{1}=-0.87272061 \equiv \omega_{d},
\end{aligned}
$$

while in case 3 they are

$$
\begin{aligned}
& \omega_{0}-\omega_{1}=-0.78809986 \equiv \omega_{e}, \\
& \omega_{0}+\omega_{1}=-0.88964476 \equiv \omega_{f} .
\end{aligned}
$$

\section{B. Soliton solutions}

It is well known $[25,33]$ that the standard DNLS equation (21) possesses multiple branches of localized solutions which are parametrized by the frequency detuning outwards from the phonon band. Multiple branches of the stationary localized solutions were also obtained in a different model in the presence of both linear and nonlinear lattices [19]. This allows one to conjecture that any of the lattices introduced in Sec. II should also possess multiple branches of the localized solutions and kinks (discrete dark solitons). Although a thorough study of each of the cases is by itself a nontrivial problem that requires lengthy considerations, to present a panoramic view of the possible nonlinear modes in the above lattices, in the present paper we restrict our study to some representative examples, referring, in most cases, to the lowest branches.

Case $2\left(W_{2}=0\right)$. For the nonlinearity of case 2, using the method described in Sec. V A, we obtain examples of localized solutions of Eq. (35), namely, pulse, staggered pulse,
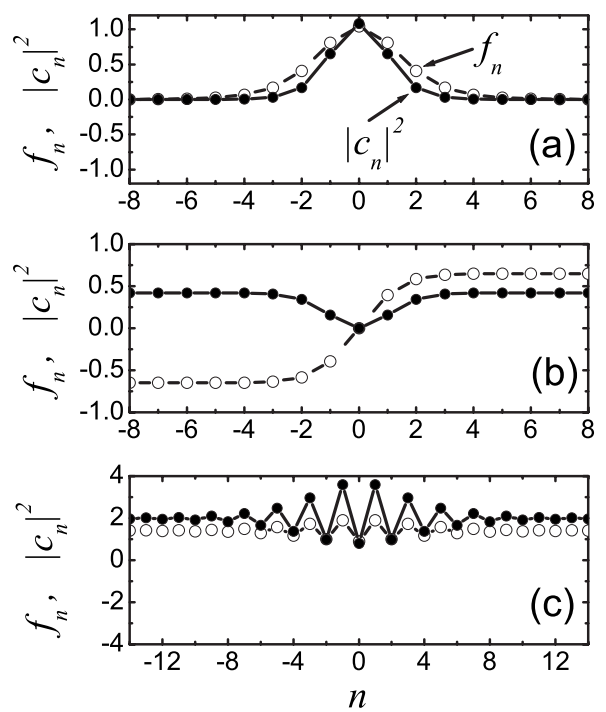

FIG. 2. Equilibrium soliton solutions found in case $2\left(W_{2}=0\right)$ : (a) pulse, (b) kink, and (c) antidark structure with oscillatory wave structure around the background, hereafter termed wave. On-site structures are presented here, but intersite ones can also be constructed. Solutions in (a) and (b) can be stable but we were unable to find a stable structure of the form presented in (c) (see Sec. V C). Model parameters corresponding to panels (a)-(c) are depicted by dots in Fig. 3 marked by the capital letters $A-C$, respectively. Parameters are (a) $W_{1}=-0.0112, \omega=-0.99$, (b) $W_{1}=0.0112, \omega=-0.9$, and (c) $W_{1}=0.0112, \omega=-0.75$.

kink, staggered kink, wave (an antidark structure in the form of an oscillatory wave on a nonzero background), and staggered wave. The nonstaggered solutions are presented in Fig. 2 for the parameters: (a) $W_{1}=-0.0112, \omega=-0.99$, (b) $W_{1}$ $=0.0112, \omega=-0.9$, (c) $W_{1}=0.0112, \omega=-0.75$. The corresponding staggered solutions can be constructed using the staggering transformation, described above. On-site equilibrium structures are shown but one can also obtain the intersite ones.

In Fig. 3, the regions of existence of various solutions are indicated on the parameter plane $\left(W_{1}, \omega\right)$. Solutions presented in Figs. 2(a)-2(c) have parameters shown by dots marked by the capital letters $A-C$, respectively. Recall that pulses can exist in the two frequency ranges $\omega<\omega_{b}$ and $\omega$ $>\omega_{a}$ for any $W_{1}$. However, they were found only for $W_{1}$ $<0$, while for $W_{1}>0$ the iterations initiated by the tail solution resulted in unbounded structures. In the portion of the plane with $W_{1}>0$ and $\omega_{b}<\omega<\omega_{c}$ kinks [see Fig. 2(b)], staggered waves were obtained. On the other hand, in the portion with $W_{1}>0$ and $\omega_{d}<\omega<\omega_{a}$, we could construct staggered kinks and waves [see panel (c) of Fig. 2]. It is important to note that $|z|$ is close to 1 near the lines $\omega=\omega_{a}$ and $\omega=\omega_{b}$, where pulses, kinks, and waves were found to be wide; $z$ diverges (or vanishes) at $\omega=\omega_{c}$ for staggered carrier field and at $\omega=\omega_{d}$ for nonstaggered carrier field; in the range of $\omega_{d}<\omega<\omega_{c}, z$ is always negative and close to 0 , resulting in rapidly oscillating or sharply localized (and typically unstable) solutions found from the tail construction.

Case $3\left(W_{1}=0\right)$. In case 3 , three examples of the nonstaggered stationary soliton solutions presented in Fig. 4 were 


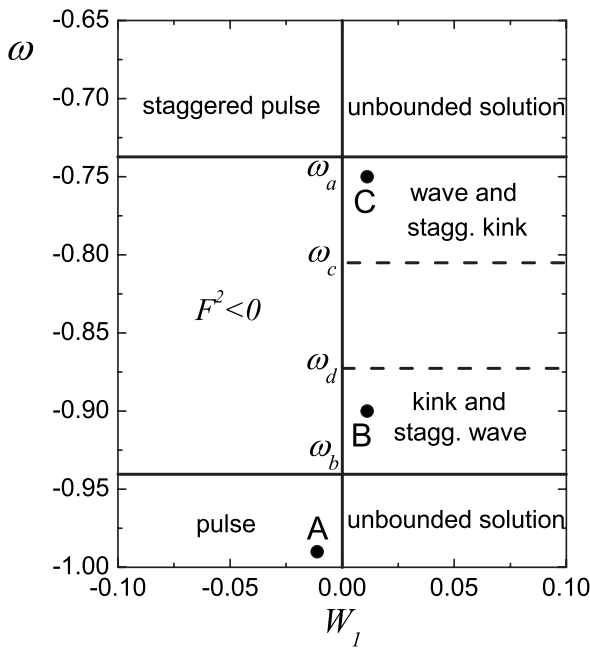

FIG. 3. Parameter plane $\left(W_{1}, \omega\right)$ in case $2\left(W_{2}=0\right)$ with indicated regions of existence of three types of solitons shown in Fig. 2 and their staggered analogs. The pulse solution exists for $W_{1}<0$ and $\omega<\omega_{b}$, while the staggered pulse exists for $W_{1}<0$ and $\omega$ $>\omega_{a}$. The kink and staggered wave can exist for $W_{1}>0$ and $\omega_{b}$ $<\omega<\omega_{c}$, while the staggered kink and the wave can exist for $W_{1}$ $>0$ and $\omega_{d}<\omega<\omega_{a}$. The values of special frequencies $\omega_{a}$ to $\omega_{d}$ are given by Eqs. (46) and (47).

found. Shown are (a) pulse, (b) kink, and (c) kink with oscillatory tail, referred to as oscillatory kink. On-site structures are presented here, but intersite ones can also be constructed. Model parameters corresponding to panels (a)-(c)
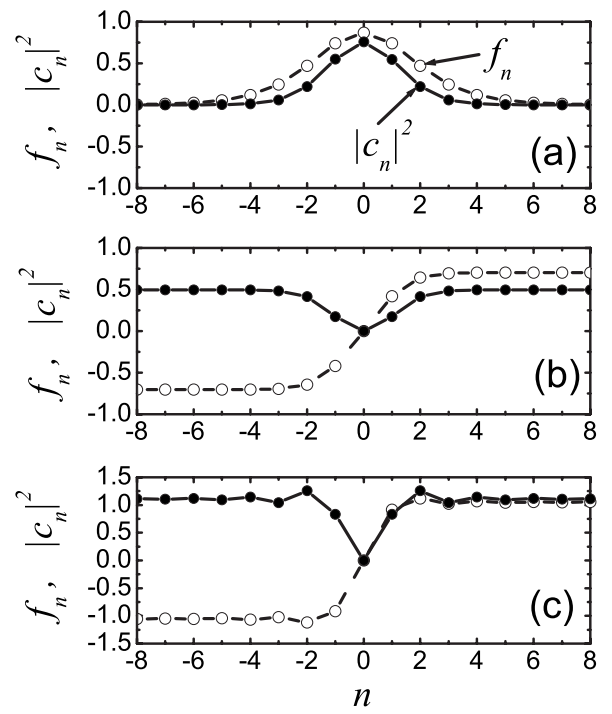

FIG. 4. Equilibrium soliton solutions obtained in case 3 with $W_{1}=0$ : (a) pulse, (b) kink, and (c) kink with oscillatory tail, called oscillatory kink. On-site structures are presented here, but intersite ones can also be constructed. All these solutions can be stable, as will be shown in Sec. V C. Model parameters corresponding to panels (a)-(c) are depicted by dots in Fig. 5 marked by the capital Latin letters $A-C$, respectively. Parameters are (a) $W_{2}=-0.0136$, $\omega=-0.9704$, (b) $W_{2}=0.0136, \omega=-0.9$, and (c) $W_{2}=0.0136, \omega=$ -0.85 .

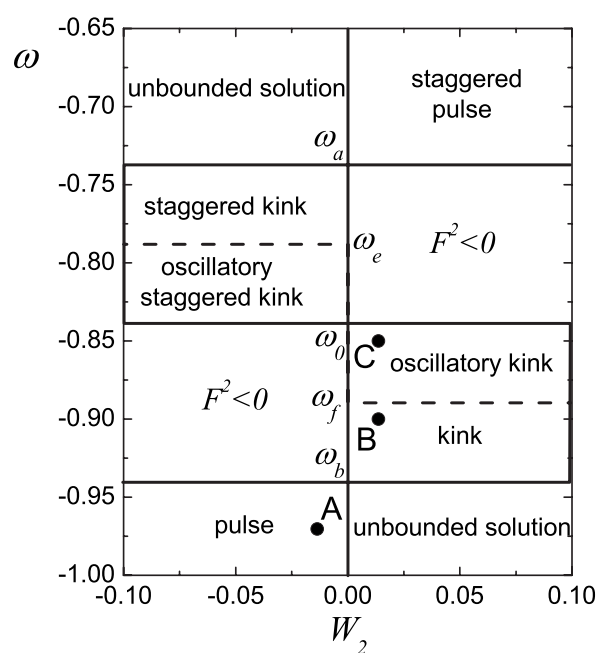

FIG. 5. Parameter plane $\left(W_{2}, \omega\right)$ in case $3\left(W_{1}=0\right)$ with indicated regions of existence of three types of solitons shown in Fig. 4 and their staggered analog. The pulse exists for $W_{2}<0$ and $\omega$ $<\omega_{b}$, while the staggered pulse exists for $W_{2}>0$ and $\omega>\omega_{a}$. The kink exists for $W_{2}>0$ and $\omega_{b}<\omega<\omega_{f}$, while the staggered kink exists for $W_{2}<0$ and $\omega_{e}<\omega<\omega_{a}$. Finally, the oscillatory kink exists for $W_{2}>0$ and $\omega_{f}<\omega<\omega_{0}$, while the oscillatory staggered kink exists for $W_{2}<0$ and $\omega_{0}<\omega<\omega_{e}$. Values of special frequencies are given by Eqs. (46) and (48).

of Fig. 4 are depicted by dots in Fig. 5 marked by the capital letters $A-C$, respectively. Parameters for the nonstaggered solutions are (a) $W_{2}=-0.0136, \omega=-0.9704$, (b) $W_{2}=0.0136$, $\omega=-0.9$, and (c) $W_{2}=0.0136, \omega=-0.85$, while the corresponding staggered solutions, as in case 2, can be obtained using the staggering transformation.

The pulse tail solution (with $z>0$ ) exists for $\omega<\omega_{b}$ but the pulse itself exists in this region only for $W_{2}<0$, while for positive $W_{2}$ we obtained unbounded solutions. Similarly, the staggered pulse tail solution (with $z<0$ ) exists for $\omega>\omega_{a}$ but the staggered pulse itself exists in this region only for $W_{2}$ $>0$, while negative $W_{2}$ leads to unbounded solutions.

The kink exists for $W_{2}>0$ and $\omega_{b}<\omega<\omega_{f}$, while the staggered kink exists for $W_{2}<0$ and $\omega_{e}<\omega<\omega_{a}$. Finally, the oscillatory kink exists for $W_{2}>0$ and $\omega_{f}<\omega<\omega_{0}$, while the oscillatory staggered kink exists for $W_{2}<0$ and $\omega_{0}<\omega$ $<\omega_{e}$.

In Figs. 6 and 7 we contrast the behavior of pulses in cases 2 and 3, respectively. In both figures (a) shows the space-time evolution of $\left|c_{n}(t)\right|^{2}$, while (b) shows the time variation of the norm of the central particles. On purpose, we did not optimize the choice of $C_{ \pm}$to get equilibrium on-site or intersite solutions. As a result, the ensuing profiles are nonstationary and, due to the presence of the Peierls-Nabarro potential, they oscillate in the vicinity of stable configurations. It turns out that in case 2 (case 3 ) the intersite (on-site) configuration is stable. This conclusion will be confirmed in Sec. V C. This illustrates the interesting phenomenon of potential inversion of stability (cf. [22]) in comparison with the standard DNLS mode [7]. Parameters in Fig. 6 are $W_{1}$ $=-0.012, W_{2}=0$, and $\omega=-0.99$, which corresponds to the point $A$ in Fig. 3. Parameters in Fig. 7 are $W_{1}=0, W_{2}$ 

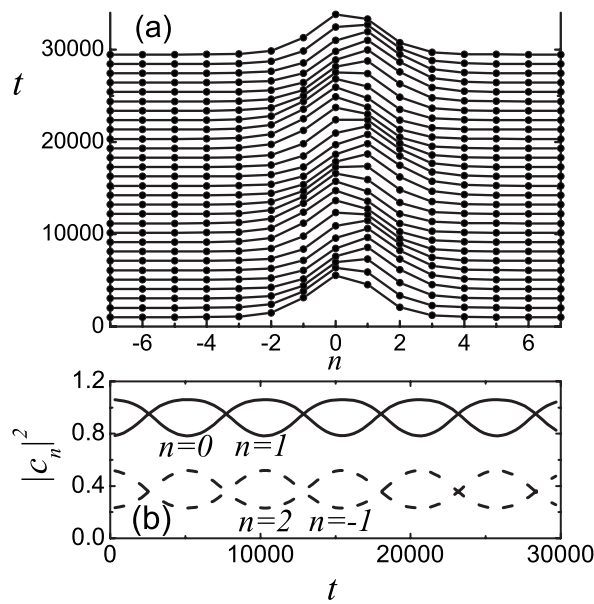

FIG. 6. Pulse in case $2\left(W_{2}=0\right)$. (a) Space-time evolution of $\left|c_{n}(t)\right|^{2}$ showing the dynamics of the pulse placed at $t=0$ asymmetrically with respect to the lattice. The pulse undergoes periodic oscillations in the vicinity of the stable intersite configuration. (b) Time variation of the norm of the four central particles. Parameters: $W_{1}$ $=-0.012, W_{2}=0$, and $\omega=-0.99$; these correspond to the point $A$ in Fig. 3.

$=-0.0136$, and $\omega=-0.9704$, which corresponds to the point $A$ in Fig. 5.

Similar results for dark solitons are presented in Fig. 8 (kink in case 2) and Fig. 9 (kink with oscillatory tail in case 3). One can see that in both cases the on-site structures are stable and this will be confirmed in Sec. V C. Parameters in Fig. 8 are $W_{1}=0.012, W_{2}=0$, and $\omega=-0.9$, which corresponds to the point $C$ in Fig. 3. Parameters in Fig. 9 are $W_{1}=0, W_{2}=0.0136$, and $\omega=-0.88$.

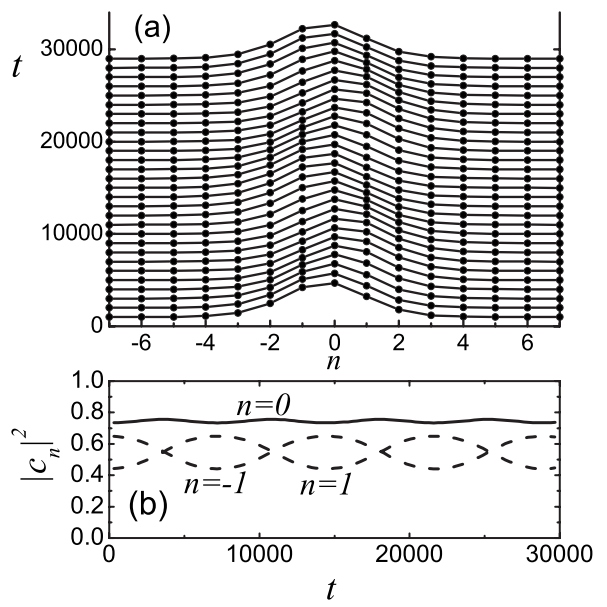

FIG. 7. Pulse in case $3\left(W_{1}=0\right)$. (a) Space-time evolution of $\left|c_{n}(t)\right|^{2}$ showing the dynamics of the pulse placed at $t=0$ asymmetrically with respect to the lattice. The pulse undergoes periodic oscillations in the vicinity of the stable on-site configuration. (b) Time variation of the norm of the central particle and its two nearest neighbors. Parameters: $W_{1}=0, W_{2}=-0.0136$, and $\omega=-0.9704$; these correspond to the point $A$ in Fig. 5 .
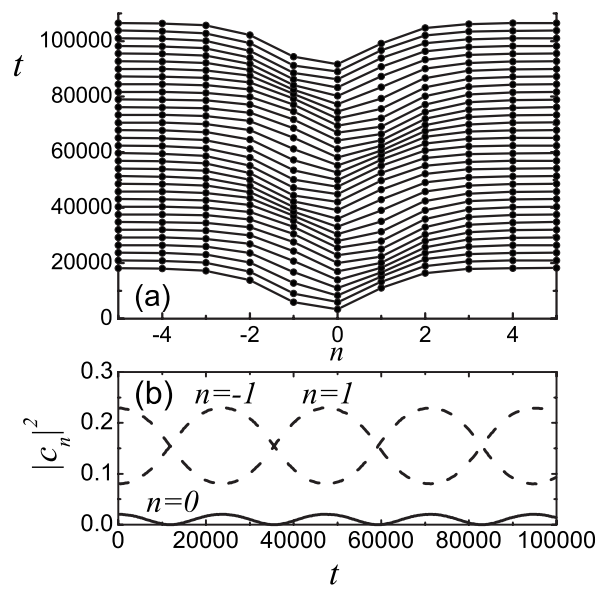

FIG. 8. Kink in case $2\left(W_{2}=0\right)$. (a) Space-time evolution of $\left|c_{n}(t)\right|^{2}$ showing the dynamics of the kink placed at $t=0$ asymmetrically with respect to the lattice. The kink undergoes periodic oscillations in the vicinity of the stable on-site configuration. (b) Time variation of the norm of the central particle and its two nearest neighbors. Parameters: $W_{1}=0.012, W_{2}=0$, and $\omega=-0.9$, which corresponds to the point $B$ in Fig. 3 .

\section{Stability of soliton solutions}

Let us now study the stability of the stationary soliton solutions of the form of Eq. (36) described in Sec. V B. We consider the following perturbed form of the solutions:

$$
c_{n}(t)=\left[f_{n}+\epsilon_{n}(t)\right] \mathrm{e}^{-i \omega t},
$$

where the small complex perturbation $\epsilon_{n}(t)$ is expressed as follows:
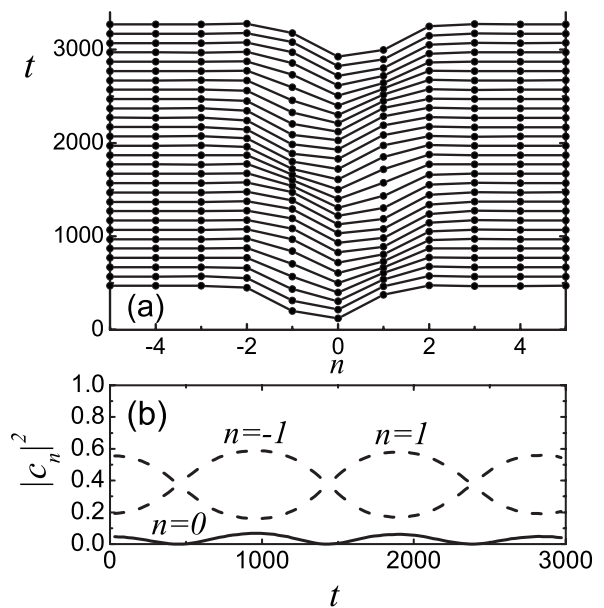

FIG. 9. Kink with oscillatory tail in case $3\left(W_{1}=0\right)$. (a) Spacetime evolution of $\left|c_{n}(t)\right|^{2}$ showing the dynamics of the kink with oscillatory tail placed at $t=0$ asymmetrically with respect to the lattice. The kink with oscillatory tail undergoes periodic oscillations in the vicinity of the stable on-site configuration. (b) Time variation of the norm of the central particle and its two nearest neighbors. Parameters: $W_{1}=0, W_{2}=0.0136, \omega=-0.88$. The kink with oscillatory tail has frequency close to $\omega_{f}=-0.8896$ and it becomes unstable far from this line (see Fig. 15). 

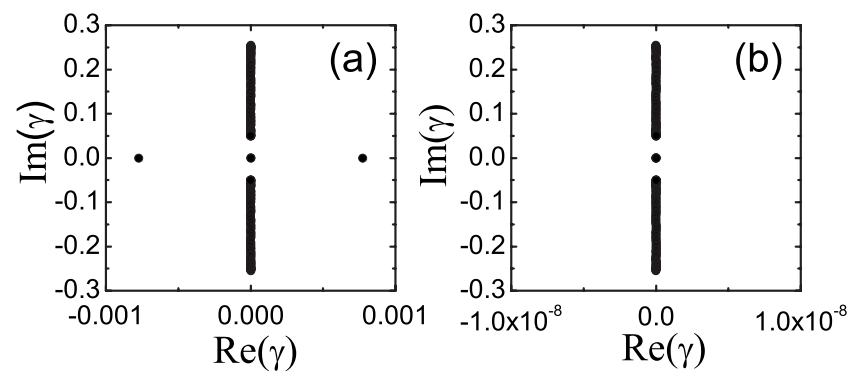

FIG. 10. Spectra of (a) unstable on-site and (b) stable intersite pulses. The parameters (case 2): $W_{1}=-0.012, W_{2}=0$, and $\omega=$ -0.99 correspond to the point $A$ in Fig. 3 .

$$
\epsilon_{n}(t)=a_{n}(t)+i b_{n}(t) .
$$

Substituting Eq. (49) into Eq. (10) we find that $\epsilon_{n}(t)$ is governed by the following linearized equation:

$$
\begin{aligned}
i \dot{\epsilon}_{n}= & \left(\omega_{0}-\omega\right) \epsilon_{n}+\omega_{1}\left(\epsilon_{n-1}+\epsilon_{n+1}\right)+W_{1}\left[f_{n-1}^{2} Z_{n-1}\right. \\
& \left.+2 f_{n}\left(f_{n-1}+f_{n+1}\right) Z_{n}+f_{n+1}^{2} Z_{n+1}+f_{n}^{2}\left(Z_{n-1}+Z_{n+1}\right)\right] \\
& +W_{2}\left[2 f_{n} f_{n-1} Z_{n-1}+\left(f_{n-1}^{2}+f_{n+1}^{2}\right) Z_{n}+2 f_{n} f_{n+1} Z_{n+1}\right],
\end{aligned}
$$

where $Z_{n}=3 a_{n}+i b_{n}$. Then, separating real and imaginary parts of Eq. (51) we derive the following system:

$$
\left(\begin{array}{c}
\dot{\mathbf{b}} \\
\dot{\mathbf{a}}
\end{array}\right)=\left(\begin{array}{cc}
0 & \mathbf{K} \\
\mathbf{J} & 0
\end{array}\right)\left(\begin{array}{l}
\mathbf{b} \\
\mathbf{a}
\end{array}\right),
$$

where vectors $\mathbf{a}$ and $\mathbf{b}$ contain $a_{n}$ and $b_{n}$, respectively, while the nonzero coefficients of matrices $\mathbf{K}$ and $\mathbf{J}$ are given by

$$
\begin{gathered}
K_{n, n-1}=-\omega_{1}-3 W_{1}\left(f_{n-1}^{2}+f_{n}^{2}\right)-6 W_{2} f_{n-1} f_{n}, \\
K_{n, n}=\omega-\omega_{0}-6 W_{1} f_{n}\left(f_{n-1}+f_{n+1}\right)-3 W_{2}\left(f_{n-1}^{2}+f_{n+1}^{2}\right), \\
K_{n, n+1}=-\omega_{1}-3 W_{1}\left(f_{n}^{2}+f_{n+1}^{2}\right)-6 W_{2} f_{n} f_{n+1}, \\
J_{n, n-1}=\omega_{1}+W_{1}\left(f_{n-1}^{2}+f_{n}^{2}\right)+2 W_{2} f_{n-1} f_{n}, \\
J_{n, n}=\omega_{0}-\omega+2 W_{1} f_{n}\left(f_{n-1}+f_{n+1}\right)+W_{2}\left(f_{n-1}^{2}+f_{n+1}^{2}\right), \\
J_{n, n+1}=\omega_{1}+W_{1}\left(f_{n}^{2}+f_{n+1}^{2}\right)+2 W_{2} f_{n} f_{n+1} .
\end{gathered}
$$

In the above expressions, $n=1, \ldots, \mathcal{N}$, where $\mathcal{N}$ is the number of lattice points. For pulses and kinks, we used periodic and antiperiodic boundary conditions, respectively.

A stationary solution is characterized as linearly stable if and only if the eigenvalue problem

$$
\left(\begin{array}{cc}
0 & \mathbf{K} \\
\mathbf{J} & 0
\end{array}\right)\left(\begin{array}{l}
\mathbf{b} \\
\mathbf{a}
\end{array}\right)=\gamma\left(\begin{array}{l}
\mathbf{b} \\
\mathbf{a}
\end{array}\right)
$$

results in nonpositive real parts of all eigenvalues $\gamma$.

The results of the stability analysis for the equilibrium structures reported in Sec. V B are presented in Figs. 10-12 for case 2 and in Figs. 13-15 for case 3. The presented spectra contain (i) the vibration frequencies of the homogeneous
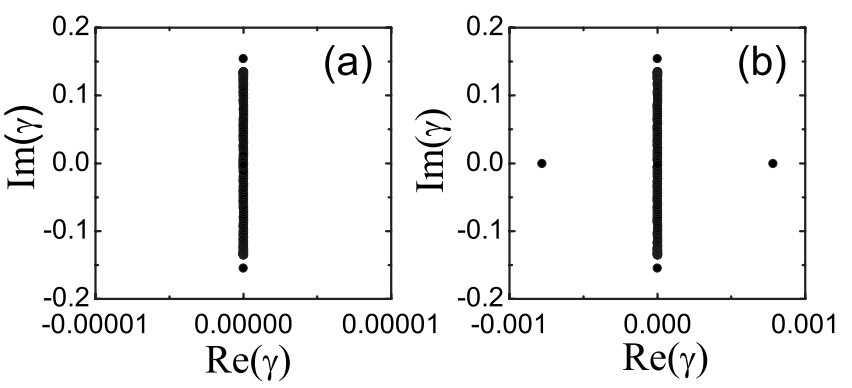

FIG. 11. Spectra of (a) stable on-site and (b) unstable intersite kinks. The parameters (case 2): $W_{1}=0.012, W_{2}=0$, and $\omega=-0.9$ correspond to the point $B$ in Fig. 3 .

background given by Eq. (26); (ii) a pair of zero-frequency modes corresponding to the invariance with respect to the phase shift; (iii) they also can include soliton internal modes falling outside the phonon band, see, e.g., Fig. 14(a). As was already mentioned, the spectra of stable structures do not possess eigenvalues with positive real parts, while those of the unstable ones have such eigenvalues. Now we turn to the discussion and comparison of the spectra in cases 2 and 3.

Case $2\left(W_{2}=0\right)$. Spectra of the on-site and intersite pulses are presented in Figs. 10(a) and 10(b), respectively. Interestingly, the intersite configuration is stable while the on-site one is unstable. This type of instability is typical for the discrete system with Peierls-Nabarro potential, although in the standard cubic onsite nonlinearity case, the results are entirely reversed in comparison to the present case [7] (e.g., the on-site pulse is stable, while the intersite features a real eigenvalue pair). This indicates that case 2 nonlinearity results in the shape of the Peierls-Nabarro potential having a maximum (minimum) for the on-site (intersite) pulses. Notice that as discussed in [22], such inversions of stability may occur in such generalized models, upon varying their relevant parameters (such as $W_{1}$ in the present case). We will see that for case 3 nonlinearity the situation for the pulse is exactly the opposite. The parameters used in this case are $W_{1}=-0.012, W_{2}=0, \omega=-0.97$, corresponding to the point $A$ in Fig. 3.

Figure 11 shows results for the kink structures: the on-site kink in (a) is stable while the intersite one in (b) is unstable. Here the location of maxima and minima of the PeierlsNabarro potential is the same as in the classical discretiza-
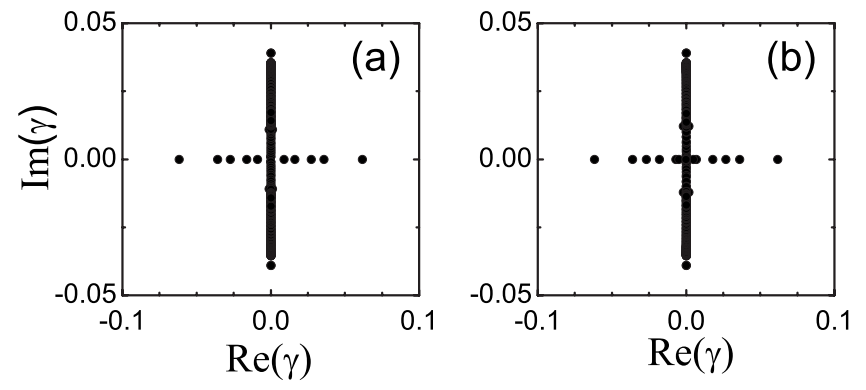

FIG. 12. Spectra of (a) unstable on-site and (b) unstable intersite wave. The parameters (case 2): $W_{1}=0.012, W_{2}=0$, and $\omega=-0.75$ correspond to the point $C$ in Fig. 3 . 

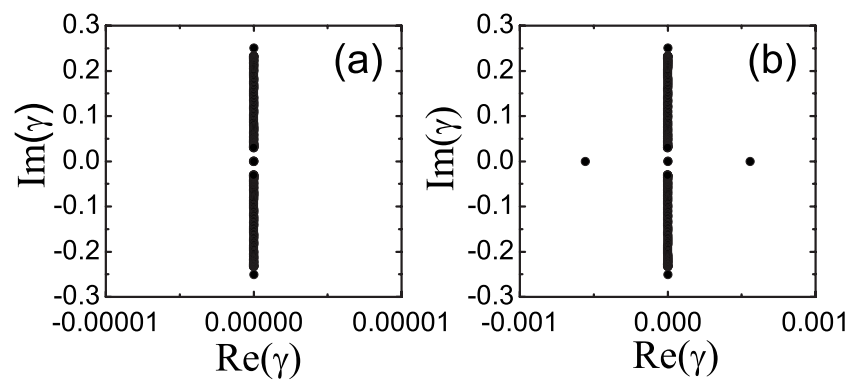

FIG. 13. Spectra of (a) stable on-site and (b) unstable intersite pulses. Oscillatory motion of the pulse in the vicinity of the on-site configuration is shown in Fig. 6. The parameters (case 3) $W_{1}=0$, $W_{2}=-0.0136, \omega=-0.9704$ correspond to the point $A$ in Fig. 5 .

tion. The parameters $W_{1}=0.012, W_{2}=0.0$, and $\omega=-0.9$ correspond to the point $B$ in Fig. 3 .

Finally, in Fig. 12 we show that (a) the on-site wave and (b) the intersite wave are both unstable. The parameters $W_{1}$ $=0.012, W_{2}=0$, and $\omega=-0.75$ correspond to the point $C$ in Fig. 3.

Case $3\left(W_{1}=0\right)$. Results of the stability analysis are presented in Figs. 13-15 for the three soliton solutions displayed in panels (a)-(c) of Fig. 4, respectively. The left panels show the spectra of the on-site structures and the right panels show the same for the corresponding intersite structures. One can see that, in contrast to case 2, where the intersite pulse was found to be stable, in case 3 the intersite structures are always unstable (this is analogous to the case of the standard cubic discrete model with the on-site nonlinearity). This indicates that in case 3 , the on-site (intersite) structures are situated in the wells (on the peaks) of the Peierls-Nabarro potential. On the other hand, panels (a) in Figs. 13-15 present purely imaginary spectra for the on-site configurations, and this indicates that, for the corresponding values of model parameters, all three types of equilibrium solutions are stable. However, in Fig. 15 we demonstrate that the on-site configuration of the oscillatory kink is stable at $\omega=0.88$ [see panel (a)] but it can become unstable, e.g., at $\omega=0.85$ [see panel (c)] with other parameters being unchanged and equal to $W_{1}=0, W_{2}=0.0136$.

\section{CONCLUSIONS}

In this work, we have illustrated the potential for formulation of a rich variety of tight-binding nonlinear lattice dy-
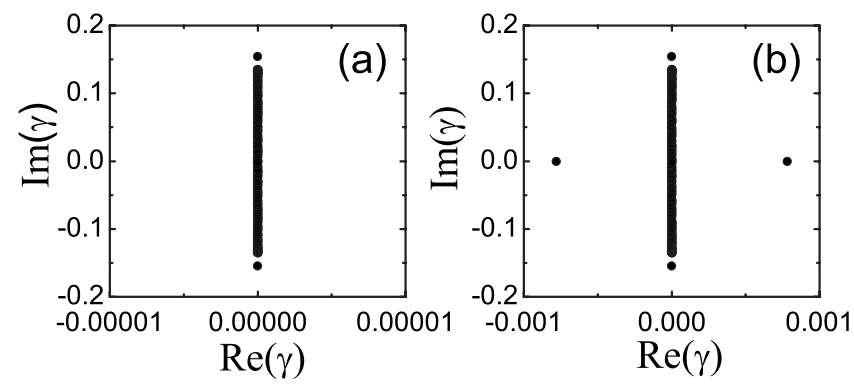

FIG. 14. Spectra of (a) stable on-site and (b) unstable intersite kinks. The parameter values (case 3) $W_{1}=0, W_{2}=0.0136$, and $\omega=-0.9$ correspond to the point $B$ in Fig. 5 .
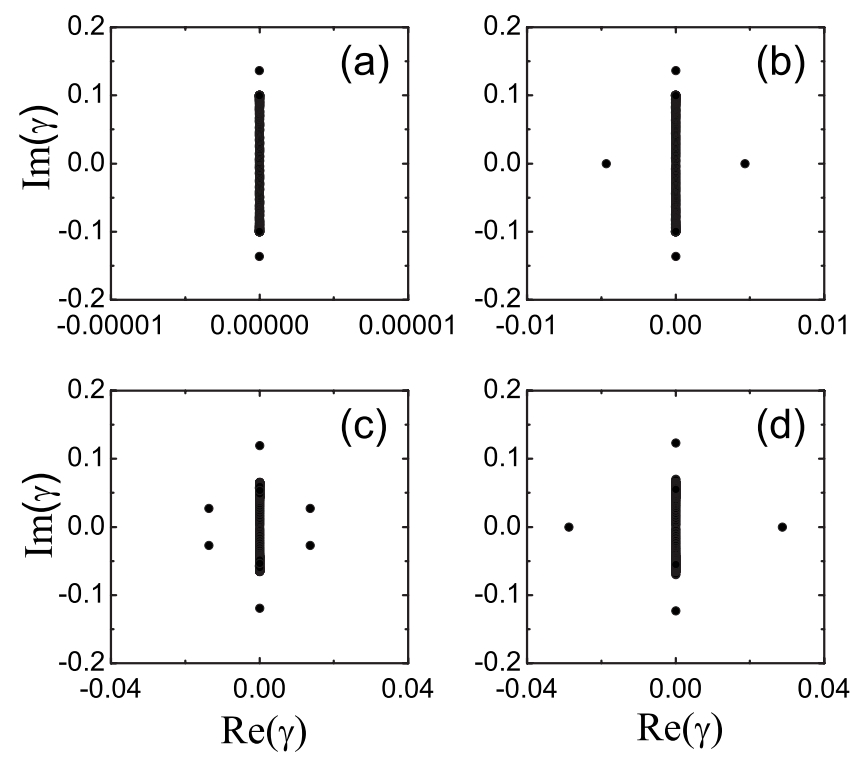

FIG. 15. The top panels show spectra of (a) stable on-site and (b) unstable intersite oscillatory kinks with $\omega=-0.88$. The bottom panels show the same for $\omega=-0.85$ when both on-site and intersite oscillatory kinks become unstable. Note that the type of instability in (c) is different from that in (d). In (c) there is a quartet of eigenvalues, while in (d) there is a pair of purely real eigenvalues. The rest of the parameters are (case 3) $W_{1}=0, W_{2}=0.0136$.

namical models, stemming from the complex interplay of linear and nonlinear periodic potentials in the nonlinear Schrödinger equation. We have examined various particular possibilities, including quasilinear models, as well as strongly nonlinear models where the nature of the coupling between the neighbors is itself nonlinear. Furthermore, in the nonlinear ones among our models, we have studied the potential for the existence and stability, as well as the dynamics of localized solutions. More specifically, we have reported that the discrete model of Eq. (35) with $W_{1} \neq 0, W_{2}=0$ (case 2 ), and $W_{1}=0$ and $W_{2} \neq 0$ (case 3 ) supports a number of localized stationary solutions depicted in Figs. 2 and 4. Interestingly, the nonlinearity of case 2 results in stable intersite pulse and stable on-site kink solutions (both in staggered and nonstaggered forms). On the other hand, the nonlinearity of the case 3 supports only on-site stable localized solutions of three different types, namely, pulses, kinks, and kinks with oscillatory tails (all in both staggered and nonstaggered forms). These results suggest that in the discrete models with nonlinear terms including interactions between nearest neighbors the profile of the Peierls-Nabarro potential can change qualitatively depending on the structure of the nonlinear terms and on the type of the coherent structure. In fact, it has been demonstrated that there exists a wide class of nonintegrable discrete models of this sort where the PeierlsNabarro potential is precisely equal to zero and equilibrium stationary solutions can be placed anywhere with respect to the lattice points [34].

At the same time, it should be mentioned that we have left open an important question about the mathematical definition of the domains of the applicability of the suggested lattice models, and more specifically of the one-band (tight- 
binding) approximation. This is a highly nontrivial question, that has not been fully addressed so far even for the more standard mapping of the NLS equation to the DNLS equation $[20,35,36]$. This question, however, goes beyond the scope of the present paper and is an important open problem. It is relevant to emphasize, in that connection, the following facts:

(1) The mapping of Eq. (1) to Eq. (5) is exact, as it is based on the expansion over the complete set of the Wannier functions. This is also the reason of the generality of the method, which can be applied to other physical systems (as a matter of fact it is implicitly applied when one uses the tightbinding approximation for the description of the light propagation in arrays of waveguides). It is particularly well suited to problems with linear (as well as nonlinear) operators with periodic coefficients.

(2) As it has been established in [20], the one-band approximation has its domain of validity, although, to the best of the authors' knowledge, it has not been established rigorously and under general conditions so far.

(3) Even in the case when the tight-binding approximation (10) does not provide the desired quantitative accuracy, it still allows for the qualitative prediction of several phenomena, including the existence, but also importantly the general stability properties of the localized modes of the system.

Another natural extension of the present work would be to consider similar types of reductions in higher dimensional settings and to examine in detail the particular localized solutions that may emerge in the resulting lattice models. In particular, higher dimensionality may offer the potential for solutions with topological charge; it would therefore be relevant to examine under what conditions such solutions may be stable and how the relevant results relate to the original continuum model.

\section{ACKNOWLEDGMENTS}

Y.V.B. was supported by the FCT Grant No. SFRH/PD/ 20292/2004. The work of P.G.K. is supported by Grants No. NSF-DMS-0505663, and No. NSF-DMS-0619492, and NSF-CAREER and by the University of Massachusetts. V.V.K. acknowledges support from Ministerio de Educación y Ciencia (MEC, Spain) under Grant No. SAB2005-0195. The work of Y.V.B. and V.V.K. was supported by the FCT and European program FEDER under Grant No. POCI/FIS/ 56237/2004. S.V.D. gratefully acknowledges the financial support provided by the Russian Foundation for Basic Research, Grant No. 07-08-12152.
[1] O. Madelung, Introduction to Solid State Theory (SpringerVerlag, Berlin, 1978).

[2] D. N. Christodoulides, F. Lederer, and Y. Silberberg, Nature (London) 424, 817 (2003); J. W. Fleischer et al., Opt. Express 13, 1780 (2005).

[3] D. N. Christodoulides and R. I. Joseph, Opt. Lett. 13, 794 (1988).

[4] H. S. Eisenberg, Y. Silberberg, R. Morandotti, A. R. Boyd, and J. S. Aitchison, Phys. Rev. Lett. 81, 3383 (1998).

[5] V. A. Brazhnyi and V. V. Konotop, Mod. Phys. Lett. B 18, 627 (2004).

[6] O. Morch and M. Oberthaler, Rev. Mod. Phys. 78, 179 (2006).

[7] P. G. Kevrekidis, K. Ø. Rasmussen, and A. R. Bishop, Int. J. Mod. Phys. B 15, 2833 (2001).

[8] A. A. Sukhorukov, Y. S. Kivshar, H. S. Eisenberg, and Y. Silberberg, IEEE J. Quantum Electron. 39, 31 (2003).

[9] R. Morandotti, U. Peschel, J. S. Aitchison, H. S. Eisenberg, and Y. Silberberg, Phys. Rev. Lett. 83, 2726 (1999).

[10] J. Meier, G. I. Stegeman, D. N. Christodoulides, Y. Silberberg, R. Morandotti, H. Yang, G. Salamo, M. Sorel, and J. S. Aitchison, Phys. Rev. Lett. 92, 163902 (2004).

[11] F. Kh. Abdullaev, B. B. Baizakov, S. A. Darmanyan, V. V. Konotop, and M. Salerno, Phys. Rev. A 64, 043606 (2001); A. Smerzi, A. Trombettoni, P. G. Kevrekidis, and A. R. Bishop, Phys. Rev. Lett. 89, 170402 (2002).

[12] F. S. Cataliotti, L. Fallani, F. Ferlaino, C. Fort, P. Maddaloni, and M. Inguscio, New J. Phys. 5, 71 (2003).

[13] Joachim Meier, Jared Hudock, Demetrios Christodoulides, George Stegeman, Y. Silberberg, R. Morandotti, and J. S. Aitchison, Phys. Rev. Lett. 91, 143907 (2003); R. L Horne, P. G. Kevrekidis, N. Whitaker, Phys. Rev. E 73, 066601 (2006).
[14] S. Suntsov, K. G. Makris, D. N. Christodoulides, G. I. Stegeman, A. Hache, R. Morandotti, H. Yang, G. Salamo, and M. Sorel, Phys. Rev. Lett. 96, 063901 (2006); Xiaosheng Wang, Anna Bezryadina, Zhigang Chen, K. G. Makris, D. N. Christodoulides, and G. I. Stegeman, ibid. 98, 123903 (2007).

[15] D. N. Neshev, T. J. Alexander, E. A. Ostrovskaya, Yu. S. Kivshar, H. Martin, I. Makasyuk, and Z. Chen, Phys. Rev. Lett. 92, 123903 (2004); J. W. Fleischer, G. Bartal, O. Cohen, O. Manela, M. Segev, J. Hudock, and D. N. Christodoulides, ibid. 92, 123904 (2004).

[16] D. E. Pelinovsky, L. Brzozowski, and E. H. Sargent, Phys. Rev. E 62, R4536 (2000).

[17] G. Fibich, Y. Sivan, and M. I. Weinstein, Physica D 217, 1 (2006); Y. Sivan, G. Fibich, and M. I. Weinstein, Phys. Rev. Lett. 97, 193902 (2006).

[18] F. Abdullaev and J. Garnier, Phys. Rev. A 72, 061605(R) (2005); G. Theocharis, P. Schmelcher, P. G. Kevrekidis, and D. J. Frantzeskakis, ibid. 72, 033614 (2005); J. Garnier and F. K. Abdullaev, ibid. 74, 013604 (2006); H. Sakaguchi and B. A. Malomed, Phys. Rev. E 72, 046610 (2005); 73, 026601 (2006); J. Belmonte-Beitia, V. M. Pérez-García, V. Vekslerchik, and P. J. Torres, Phys. Rev. Lett. 98, 064102 (2007); F. Kh. Abdullaev, A. A. Abdumalikov, and R. M. Galimzyanov, Phys. Lett. A 367, 149 (2007).

[19] Yu. V. Bludov and V. V. Konotop, Phys. Rev. A 74, 043616 (2006).

[20] G. L. Alfimov, P. G. Kevrekidis, V. V. Konotop, and M. Salerno, Phys. Rev. E 66, 046608 (2002).

[21] V. V. Konotop and S. Takeno, Phys. Rev. B 55, 11342 (1997); Physica D 113, 261 (1998).

[22] M. Öster, M. Johansson, and A. Eriksson, Phys. Rev. E 67, 
056606 (2003).

[23] M. Öster, Yu. B. Gaididei, M. Johansson, and P. L. Christiansen, Physica D 198, 29 (2004).

[24] A. Smerzi and A. Trombettoni, Phys. Rev. A 68, 023613 (2003); C. Menotti, A. Smerzi, and A. Trombettoni, New J. Phys. 5, 112 (2003).

[25] G. L. Alfimov, V. A. Brazhnyi, and V. V. Konotop, Physica D 194, 127 (2004).

[26] D. E. Pelinovsky, P. G. Kevrekidis, and D. J. Frantzeskakis, Physica D 212, 1 (2005).

[27] W. Kohn, Phys. Rev. 115, 809 (1959).

[28] Yu. S. Kivshar and M. Salerno, Phys. Rev. E 49, 3543 (1994).

[29] V. V. Konotop and S. Takeno, Phys. Rev. E 60, 1001 (1999).

[30] F. Kh. Abdullaev, S. A. Darmanyan, and J. Garnier, Prog. Opt. 44, 303 (2002).

[31] V. V. Konotop and M. Salerno, Phys. Rev. E 55, 4706 (1997); 56, 3611 (1997); V. V. Konotop, Chaos, Solitons Fractals 11, 153 (2000).

[32] G. L. Alfimov, V. V. Konotop, and M. Salerno, Europhys. Lett. 58, 7 (2002).

[33] E. W. Laedke, O. Kluth, and K. H. Spatschek, Phys. Rev. E
54, 4299 (1996)

[34] P. G. Kevrekidis, S. V. Dmitriev, and A. A. Sukhorukov, Math. Comput. Simul. 74, 343 (2007); S. V. Dmitriev, P. G. Kevrekidis, N. Yoshikawa, and D. J. Frantzeskakis, J. Phys. A 40, 1727 (2007); S. V. Dmitriev, P. G. Kevrekidis, A. A. Sukhorukov, N. Yoshikawa, and S. Takeno, Phys. Lett. A 356, 324 (2006); (see also e-print arXiv:nlin.PS/0603047, with corrected misprints); D. E. Pelinovsky, Nonlinearity 19, 2695 (2006); A. Khare, S. V. Dmitriev, and A. Saxena, J. Phys. A: Math. Theor. 40, 11301 (2007).

[35] M. J. Ablowitz and Z. H. Musslimani, Physica D 184, 276 (2003).

[36] M. J. Ablowitz, B. Prinari, and A. D. Trubatch, Discrete and Continuous Nonlinear Schrödinger Systems (Cambridge University Press, Cambridge, England, 2004).

[37] The numerical values of the Fourier coefficients, of the Wannier functions, and of the overlap integrals presented in this paper were obtained by the software developed by G. L. Alfimov.

[38] This is a variant of the shooting method used for obtaining localized modes in continuous models [5,19,25,32]. 